\title{
CICLO DIÁRIO E SEMIDIÁRIO DE PRECIPITAÇÃO NA COSTA NORTE DO BRASIL
}

\author{
CLÁUDIO MOISÉS SANTOS SILVA
}

\author{
Universidade Federal do Rio Grande do Norte (UFRN), Departamento de Física Teórica e Experimental, \\ Natal, RN, Brasil \\ claudio@dfte.ufrn.br
}

Recebido Junho de 2011 - Aceito Julho de 2012

\begin{abstract}
RESUMO
Estudou-se o ciclo diário e o ciclo semidiário da precipitação na região costeira do Norte do Brasil. Usou-se 13 anos de dados ininterruptos de precipitação estimados através do algoritmo 3B42_V6 do projeto TRMM. Os ciclos foram analisados por quadrimestres. Para obter informações sobre amplitude, fase e fração de variância de cada ciclo, utilizou-se a análise harmônica do ciclo composto no período de 24 horas para cada quadrimestre. Regiões mais próximas da costa apresentaram ciclos modulados por mecanismos oceânicos e, portanto, tendem a apresentar o máximo de precipitação no início da manhã e a noite. Regiões mais afastadas da costa apresentaram máximos às 2100 UTC (17:00 HL) em função dos mecanismos de convecção local. A região Nordeste do Pará apresentou as maiores amplitudes de precipitação. Durante o quadrimestre de janeiro a abril observou-se as maiores taxas de precipitação. Não há variações sazonais significativas na fase dos ciclos. Os dois harmônicos correspondem a cerca de $80 \%$ do total da variância diária dos dados. No final, algumas sugestões de trabalhos futuros e aplicabilidade dos dados do 3B42_V6 são discutidas.
\end{abstract}

Palavras chave: TRMM, 3B42_V6, análise harmônica.

\begin{abstract}
DIURNAL AND SEMIDIURNAL RAINFALL CYCLE OVER NORTH COASTLAND OF BRAZIL

The diurnal and semidiurnal rainfall cycles observed over the Brazilian North coastland were studied. A 13 years rainfall dataset derived by the 3B42_V6 algorithm was used. To obtain the amplitude, phase, and variance of each cycle the harmonic analysis was applied to the mean rainfall of 24 hours. Coastlands areas showed cycles modulated by oceanic mechanisms, thus the maximum rainfall was observed at early-morning and nighttime. Inland regions exhibited maximum rainfall at 2100 UTC (17:00 LST) due the local convection mechanism. The highest amplitude of precipitation was observed over the Northeast of the Pará State. The highest rainfall rates were observed during January to April period. The phase of each cycle do not presented significant seasonal variation. The diurnal and semidiurnal harmonics contributed with around $80 \%$ to total variance of the daily rainfall. Finally, some future works and applicability of the 3B42_V6 rainfall dataset were suggested.
\end{abstract}

Keywords: TRMM, 3B42_V6, harmonic analysis.

\section{INTRODUÇÃO}

Nos trópicos, dois dos principais modos de variabilidade de precipitação são o ciclo diário (24 horas) e o ciclo semidiário (12 horas). Antes do uso de dados de satélites, os estudos referentes a esses ciclos eram conduzidos a partir de alguns pontos específicos de observações, implicando em conclusões particulares para as localidades das estações meteorológicas. No caso da bacia Amazônica a pouca disponibilidade e, em muitos casos, o grande número de falhas nos dados observados à superfície via monitoramento meteorológico convencional foi sempre um entrave para o desenvolvimento de estudos sobre o ciclo diário e semidiário de precipitação.

A variabilidade diária e semidiária é manifestada em várias variáveis meteorológicas, tais como, a pressão atmosférica e o geopotencial (Dai e Wang, 1999); temperatura do ar e umidade em superfície (Chen e Wang, 1994); temperatura do ar em altitude (Wallace e Paton, 1970); Temperatura da 
Superfície do Mar (Webster et al., 1996); tamanho e cobertura de nuvens (Machado et al., 1993); vento horizontal e circulação na camada limite atmosférica (Douglas et al., 1998), balanço de radiação à superfície (Hartmann et al., 1991); frequência e intensidade de descargas atmosféricas (Boccipio et al., 2002). Trata-se, portanto, de dois dos principais modos de variabilidade da atmosfera, que são primariamente dominados pelas trocas de energia, massa e movimento próximo à superfície. Dessa forma, configuram-se como as variabilidades atmosféricas mais susceptíveis ao tipo de cobertura do solo, sendo diretamente afetadas pelos diferentes usos da terra.

Os ciclos das variáveis citadas são geralmente associados (através de relações de causa ou efeito) pelos ciclos da precipitação. Um aspecto geral sobre o ciclo de precipitação é que sobre oceanos o máximo ocorre no final da noite e início da manhã, enquanto sobre áreas continentais ocorre entre meio dia e o final da tarde. Neste sentido, Yang e Smith (2006) revisaram os principais mecanismos moduladores do ciclo diário de precipitação ao redor do globo e verificam que esse comportamento é válido para a grande escala; contudo, na escala regional e microescala, é comum a ocorrência de sinergismo entre diferentes mecanismos, principalmente sobre a América do Sul e a África. Em alguns casos, esses sinergismos podem alterar de forma significativa o ciclo diário sobre determinadas regiões, como por exemplo, na bacia Amazônica, onde é possível registrar chuva noturna em algumas localidades a partir da associação do mecanismo de brisa marítima, fontes remotas de calor e jatos em baixos níveis (Cohen et al., 1995).

A seguir descrevem-se as principais características dos mecanismos moduladores dos ciclos diário e semidiário de precipitação seguindo o trabalho de Yang e Smith (2006), que classificaram os mecanismos pelo tipo de superfície (continental ou oceânica) e pelo horário da precipitação observada (dia ou noite).

i) Continental 1: o aquecimento da superfície durante o dia induz dois efeitos diretos. O primeiro é a desestabilização estática da camada limite atmosférica, devido aos fluxos turbulentos de calor sensível e de calor latente. A intensidade da instabilização e o conteúdo de umidade disponível na atmosfera são responsáveis pela geração de células convectivas no final da tarde sobre áreas continentais (Ogura e Takahashi, 1971). O segundo efeito é a formação de circulações térmicas diretas do tipo brisa marítima e terrestre em função do aquecimento diferencial de superfícies (Pielke, 1974), que também induzem precipitação sobre regiões costeiras;

ii) Continental 2: a formação de precipitação intensa sobre regiões montanhosas é associada ao levantamento forçado de ar, que produz tempestades tanto de dia quanto à noite (Tripoli e Cotton, 1989);

iii) Continental 3: o aquecimento da superfície e, por consequência, aumento da convergência na baixa troposfera durante o dia, induz um aumento da turbulência mecânica. Assim, o número de Richardson (Ri), que mede a razão entre a turbulência gerada termicamente e a turbulência gerada mecanicamente, diminui até alcançar um valor crítico $(\approx 0,25)$, abaixo deste limiar processos convectivos são iniciados (Wallace, 1975);

iv) Continental 4: Silva Dias et al. (1987) utilizam as equações linearizadas do modelo de água rasa e com aproximação do plano $\beta$ equatorial para verificar a influência de uma forçante térmica, com ciclo diário bem definido, na circulação tropical. Esta forçante gera padrões de divergência em grande escala nos altos níveis da atmosfera, que se propagam para regiões vizinhas, influenciando o horário de ocorrência da precipitação;

v) Continental 5: a maré barométrica, induzida pelo aquecimento solar durante o dia e pela atração gravitacional lunar à noite, organiza padrões de convergência de massa em escala planetária, que modulam um ciclo semi diário (ciclo de 12 horas) de precipitação (Lindzen, 1978);

vi) Continental 6: o resfriamento dos topos de nuvens via perda de energia por processos radiativos altera o gradiente vertical de temperatura (lapse rate) causando instabilidade, e consequente precipitação durante a noite. Embora tenha sido observado sobre regiões tropicais, este mecanismo é mais frequente sobre regiões oceânicas (Dai, 2001);

vii) Oceânico 1: o máximo de precipitação observado no final da noite ocorre devido ao resfriamento do topo de nuvens e a consequente diminuição (em valor absoluto) do lapse rate atmosférico (Randall et al., 1991). Este processo é similar ao mecanismo Continental 6. Entretanto, alguns autores (Xu e Emanuel, 1989) criticam este mecanismo, pois dados observacionais não mostram diferenças significativas entre o lapse rate atmosférico observado durante o dia e durante a noite sobre os oceanos;

viii) Oceânico 2: de acordo com Gray e Jacobson (1977) a convecção noturna sobre oceanos é modulada pela variação diária do campo de divergência horizontal, a qual é gerada pelo aquecimento radiativo diferencial entre regiões com e sem nebulosidade;

ix) Oceânico 3: este mecanismo é associado à intensificação de ciclones tropicais durante o período noturno. $\mathrm{O}$ resfriamento da região da bigorna das nuvens do ciclone propaga ondas de gravidade da porção inferior para a superior. A energia transmitida dentro das nuvens durante este processo de propagação das ondas de gravidade funciona como fonte de energia para o ciclone (Tripoli, 1992);

x) Oceânico 4: o aquecimento diurno da TSM desestabiliza a atmosfera através dos fluxos de calor sensível e de calor latente, provocando um máximo de precipitação no final da tarde (Sui et al., 1997);

xi) Oceânico 5: a atividade convectiva sobre o continente tende a gerar ondas de gravidade, que se propagam na vertical 
e na horizontal, principalmente em regiões costeiras, gerando atividade convectiva sobre os oceanos (Mapes et al., 2003a, b; Warner et al., 2003).

Especificamente sobre o norte do Brasil, o ciclo diário da precipitação foi investigado através de medidas de pluviômetros durante o período de 1961 a 1970 (Kousky, 1980). Localidades ao longo da costa apresentaram o máximo de precipitação durante a noite, devido aos efeitos de brisa marítima, que se reflete em convergência na Camada Limite Atmosférica. Este autor também observou, que a sazonalidade do ciclo diário é mais pronunciada sobre áreas costeiras. Neste sentido, sítios localizados na ilha de Marajó exibem máximos à noite durante os meses chuvosos (Janeiro a Maio) e máximos durante o dia nos meses considerados secos (Junho a Setembro). Souza e Rocha (2006), usando cinco anos de dados coletados em outro ponto do litoral do Estado do Pará (município de Bragança), também verificaram que durante os meses de Janeiro a Maio o ciclo diário de precipitação exibe um máximo nos horários da manhã e madrugada.

Com dados do Geostationary Operational Environmental Satellite Precipitation Index (GPI), Mapes et al. (2003a) estudaram o ciclo diário da precipitação sobre a Amazônia para o período de agosto de 1998 a julho de 2000. Grande parte da convecção ocorre na forma de pequenos aglomerados convectivos no interior da bacia. Porém, formações de mesoescala em formato de linhas (Linhas de Instabilidade, Cohen et al., 1995), ao longo da costa, apresentam propagação de 2 a 3 dias para Oeste e são responsáveis pela modulação do ciclo diurno.

Barbosa et al. (2006) realizou um monitoramento das perturbações convectivas formadas sob a costa Norte do Brasil, através dos dados do International Satellite Cloud Climatology Project (ISCCP), durante os anos de 1984 a 1998, identificando três tipos de sistemas costeiros: (i) perturbações pontuais identificadas em apenas um instante e não mais no horário seguinte com horário preferencial de ocorrência às 1800 UTC; (ii) perturbações não pontuais identificadas em pelo menos dois horários, apresentando deslocamento inferior a $150 \mathrm{~km}$, formadas às 2100 UTC e que duram pelo menos 6 horas; (iii) perturbações não pontuais com deslocamento acima de $150 \mathrm{~km}$, maior extensão horizontal e apresentam ciclo de vida entre 6 e 12 horas. O deslocamento preferencial destes sistemas é para Oeste, sendo o mecanismo de brisa marítima o principal responsável pela formação deles, enquanto as forçantes dinâmicas de grande escala determinam sua intensificação e os limites de propagação.

Com o início das atividades do projeto Tropicall Rainfall Measuring Mission (TRMM) (Simpson et al., 1988) foi possível obter informações da estrutura tridimensional da atividade convectiva sobre as áreas tropicais e subtropicais do globo. Com isso, desenvolveram-se vários estudos e possibilidades de aplicações dos produtos derivados do TRMM. Os produtos que geram dados de precipitação, como é o caso do algoritmo
3B42_V6, passaram a fornecer informações em uma grade horizontal com espaçamento de $25 \mathrm{~km}$ e amostragem temporal de 3 horas. Logo, de posse desses dados, tornou-se possível estudar escalas atmosféricas desde o ciclo semidiário até a variabilidade inter anual.

Assim, a motivação principal deste artigo é a possibilidade de se realizar um estudo com 13 anos de dados de precipitação em alta amostragem espaço-temporal ( $25 \mathrm{~km}$ x $25 \mathrm{~km}$ e 3 horas) sobre parte da Amazônia. Especificamente, escolheu-se a região que será chamada a partir daqui de costa Norte do Brasil (Figura 1), por se tratar de uma área com alguns estudos observacionais prévios (Kousky, 1980; Souza e Rocha, 2006), que servirão de base para a discussão dos resultados aqui apresentados. Além disso, é uma área onde reconhecidamente o mecanismo de brisa marítima modula a formação de sistemas convectivos em forma de Linhas de Instabilidade (Cohen et al., 1995), impondo ciclos diário e semidiário bem definidos ao longo da costa e no interior do continente. Pretende-se identificar as regiões onde cada ciclo é dominante em termos do total de variância explicada, destacando-se as variações sazonais de cada ciclo em diferentes sub áreas.

\section{MATERIAIS E METODOLOGIA}

\subsection{Dados do algoritmo 3B42 V6}

A precipitação através do algoritmo 3B42_V6 é obtida a partir da Técnica de Análise de Precipitação de Multi-satélites

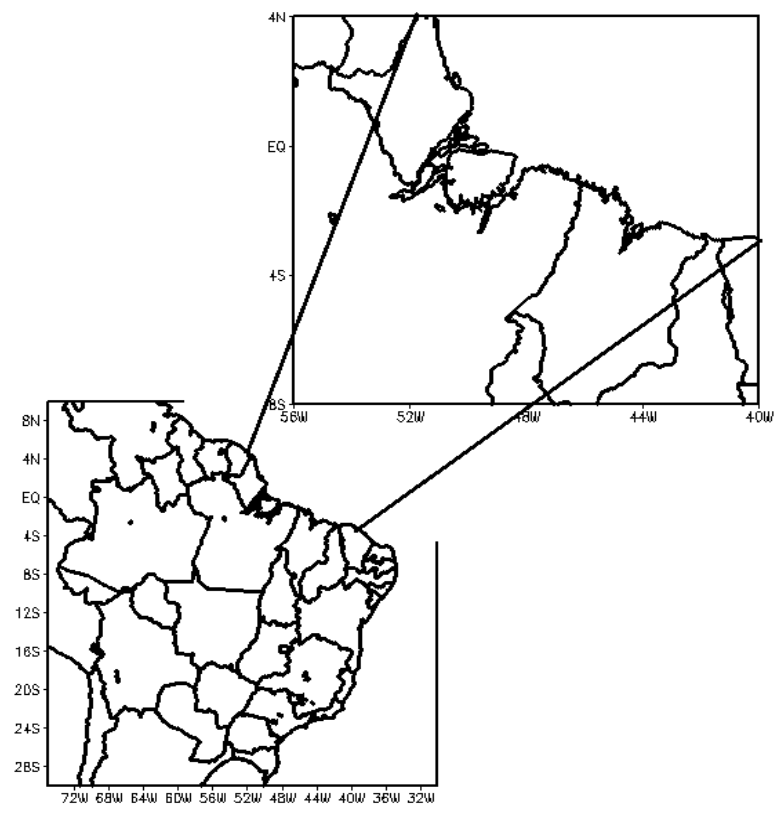

Figura 1 - Mapa do Brasil, destacando-se a área que foi chamada de Costa Norte do Brasil. 
(TMPA) (TRMM Multisatellite Precipitation Analysis) (Huffman et al., 2007). Os dados cobrem a faixa latitudinal de $50^{\circ} \mathrm{S}$ a $50^{\circ} \mathrm{N}$ e longitudinal de 0 a $360^{\circ}$. Estão dispostos em uma grade regular cujo espaçamento é de $0,25^{\circ} \mathrm{e}$ a amostragem temporal é a cada 3 horas, sendo a primeira estimativa de precipitação fornecida às 0000 UTC. O período de dados usados neste artigo foi de $0000 \mathrm{Z}$ de $1^{\circ}$ de janeiro de 1998 a $2100 \mathrm{Z}$ de 30 de Abril de 2011.

\subsection{Análise harmônica}

A análise harmônica consiste em representar uma função periódica em somas algébricas de componentes de senos e cossenos. Para o período de 24 horas, o primeiro harmônico corresponde ao ciclo diário, enquanto o segundo harmônico descreve o ciclo semidiário. Com o uso da análise harmônica é possível identificar as seguintes características de cada ciclo: i) a amplitude de cada harmônico, que é uma medida da diferença entre os valores máximos e mínimos alcançados por um determinado harmônico; ii) o ângulo de fase, que mostra o intervalo (em horas ou radianos) que a onda leva para sair da primeira observação e alcançar o valor máximo, logo, indica o horário do máximo de precipitação; iii) a fração de variância, que indica quanto cada harmônico contribui para o total da variância dos dados analisados. Seguindo Wilks (2006) a análise harmônica através de séries de Fourier é determinada pelas expressões:

$$
\begin{aligned}
& P(t)=\bar{P}+\sum_{k=1}^{2} c_{k} \cos \left(\omega_{k} t-\varphi_{k}\right) \\
& c_{k}=\sqrt{a_{k}^{2}+b_{k}^{2}}, \operatorname{com} a_{k}=\frac{2}{N} \sum_{j=1}^{N} P(t) \cos \left(\frac{2 \pi k t}{N}\right), \mathrm{e} \\
& b_{k}=\frac{2}{N} \sum_{j=1}^{N} P(t) \operatorname{sen}\left(\frac{2 \pi k t}{N}\right) \\
& \varphi_{k}=\tan ^{-1}\left(\frac{b_{k}}{a_{k}}\right) \text { se } a_{k}>0, \varphi_{k}=\tan ^{-1}\left(\frac{b_{k}}{a_{k}}\right) \pm \pi \text { se } a_{k}<0 \\
& \varphi_{k}=\frac{\pi}{2} \operatorname{se} a_{k}=0 \\
& \operatorname{var}=\frac{c_{K}^{2}}{2 \mathrm{~s}^{2}}
\end{aligned}
$$

Sendo $P(t)$ a precipitação estimada pelo $3 \mathrm{~B} 42 \_\mathrm{V} 6 ; \bar{P}$ a precipitação média no período de 24 horas; $\mathrm{k}$ representa os harmônicos; $\omega_{\mathrm{k}}$ e $c_{k}$ são, respectivamente, a frequência angular e a amplitude de cada harmônico; $a_{k}$ é a componente de cosseno e $b_{k}$ é a componente de seno de cada harmônico; $\mathrm{N}$ é o número de observações dentro do intervalo de 24 horas, para os dados do 3B42_V6, N=8; $\varphi_{k}$ é o ângulo de fase; var é a fração de variância explicada por cada harmônico; $s^{2}$ é a variância da série.
A análise harmônica foi aplicada para a climatologia (de 13 anos) do ciclo correspondente a cada quadrimestre. $\mathrm{O}$ primeiro quadrimestre corresponde aos meses de janeiro a abril (JFMA), o segundo aos meses de maio a agosto (MJJA) e o terceiro de setembro a dezembro (SOND). Apesar de ser mais comum, em estudos sobre climatologia da Amazônia, a separação das estações do ano por trimestres com início em dezembro, sabe-se que a migração da precipitação ocorre no sentido Sul para Norte a partir de dezembro (Marengo et al., 2001). Sendo assim, por estar na parte mais ao norte do Brasil, a região em estudo não apresenta precipitação elevada no mês de dezembro. Dessa forma, foi possível dividir as análises em quadrimestres a partir de janeiro, sendo que cada quadrimestre apresentou características relativamente distintas quanto à intensidade da precipitação, conforme será mostrado nas análises dos resultados. Como o foco deste estudo é no ciclo diário da região costeira, não será apresentada a variação sazonal (mês a mês) para toda a bacia Amazônica, que pode ser encontrada em Marengo e Nobre (2009).

\section{RESULTADOS}

\subsection{Média de 13 anos}

O ciclo diário de precipitação do quadrimestre JFMA é apresentado na Figura 2. Verifica-se que a atividade convectiva é iniciada ao longo da costa às $1200 \mathrm{UTC}(08: 00 \mathrm{HL})$, sendo mais intensa na área entre a Ilha do Marajó e o estado do Amapá. No horário seguinte a precipitação continua confinada a essas áreas, apresentando taxas máximas entre 0,8 e $1,0 \mathrm{~mm} \mathrm{~h}^{-1}$. Às 1800 UTC (14:00 HL) é estabelecida uma linha de precipitação ao longo de toda a costa, observando-se taxas de precipitação quase sempre superiores a $1,0 \mathrm{~mm} \mathrm{~h}^{-1}$. A linha de precipitação desloca-se continente adentro a partir das 2100 UTC (17:00 HL) ), provavelmente neste horário ocorra um acoplamento como a mesma direção e sentido entre a brisa marítima e os ventos alísios de leste. Sobre o estado do Amapá, que fica localizado perpendicularmente ao escoamento básico de Leste em baixos níveis, a propagação da precipitação ocorre com maior velocidade em comparação às outras regiões. Nesse sentido, a linha de precipitação sobre este estado encontra-se em uma posição mais central, em torno de $52^{\circ} \mathrm{W}$, que é cerca de $100 \mathrm{~km}$ de distância em relação à costa.

Às 0000 UTC (20:00 HL) a linha de precipitação já perdeu força sobre o Amapá, porém continua bastante ativa no Nordeste do Pará e ainda significativa, com taxas entre 0,6 e $0,8 \mathrm{~mm} \mathrm{~h}^{-1}$, sobre o estado do Maranhão. Ao mesmo tempo a precipitação sobre a maior parte da Ilha do Marajó apresenta taxas de precipitação entre 0,2 e $0,4 \mathrm{~mm} \mathrm{~h}^{-1}$. Às 0300 UTC (23:00 HL) a linha de precipitação perde força, embora seja 
remanescente um núcleo de precipitação em formato quase circular sobre o Nordeste do Pará. Nos horários seguintes, verifica-se a dissipação do núcleo de precipitação, sendo que às 0900 UTC (05:00 HL) ainda é possível observar taxas de precipitação entre 0,4 e $0,6 \mathrm{~mm} \mathrm{~h}^{-1}$ em áreas acerca de $500 \mathrm{~km}$ do litoral paraense. Nesse mesmo horário, uma nova linha de precipitação começa a se formar sobre o litoral, em função do mecanismo de brisa marítima.

Na Figura 3 mostra-se o ciclo diário de precipitação para o quadrimestre MJJA. Às 1200 UTC a atividade convectiva é iniciada, destacando-se sobre o litoral do Amapá. Há pouca evolução na localização e na intensidade da precipitação no horário seguinte, embora a região entre a Ilha do Marajó e o Amapá apresente taxas horárias entre 0,6 e $0,8 \mathrm{~mm}$ $\mathrm{h}^{-1}$. A formação da linha de precipitação ao longo da costa ocorre às 1800 UTC, semelhante ao quadrimestre JFMA; porém, apresenta-se menos intensa. Às 1700 UTC a linha de precipitação ainda está localizada nas proximidades da linha da costa, inclusive sobre o estado do Amapá, que no quadrimestre anterior apresentou maior propagação.

O núcleo de precipitação em formato quase circular também é verificado no Nordeste do Pará, porém abrange boa parte da Ilha do Marajó. Nos horários seguintes verifica-se pouca propagação da linha de precipitação e a dissipação desta linha ocorre em horários noturnos. Finalmente, no quadrimestre SOND (Figura 4), verifica-se que a linha de precipitação é iniciada às 1800 UTC, apresentando intensidade e área de
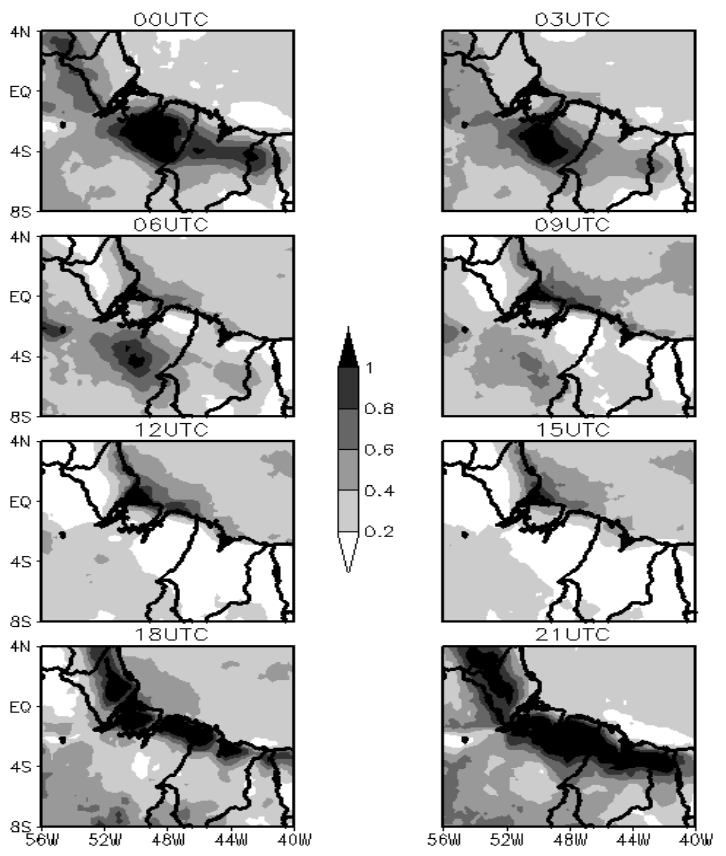

Figura 2 - Ciclo diário de precipitação sobre a costa Norte do Brasil obtida a partir da média aritmética de cada horário sobre os 13 anos de dados do 3B42 V6 para o quadrimestre JFMA. A barra no meio da figura indica taxas de precipitação expressas em mm h-1. cobertura máxima às 1700 UTC, sendo as maiores taxas verificadas sobre parte da Ilha do Marajó, Nordeste do Pará e a parte central do Amapá. Quanto à precipitação noturna, a região Nordeste do Pará apresenta um núcleo circular com intensidades entre 0,4 e $0,6 \mathrm{~mm} \mathrm{~h}^{-1}$.

\subsection{Amplitude, fase e variância}

Na Figura 5 são mostradas a amplitude, a fase e a variância explicada pelo harmônico diário. A amplitude do ciclo é maior durante o quadrimestre JFMA em comparação aos outros dois quadrimestres. A maior amplitude durante o quadrimestre JFMA é localizada sobre o Nordeste do Pará, alcançando taxas entre 0,6 e $1 \mathrm{~mm} \mathrm{~h}^{-1}$. Em ambos os quadrimestres a amplitude diminui em direção ao interior do continente, sendo sempre abaixo de $0,2 \mathrm{~mm} \mathrm{~h}^{-1}$ para áreas localizadas a mais de $450 \mathrm{~km}$ da linha da costa. A precipitação máxima, indicado pelo vetor nas figuras da primeira coluna, ocorre sempre à noite sobre o oceano. Nas localidades mais próximas da linha da costa (entre 0 e $100 \mathrm{~km}$ ), com destaque para o litoral do Amapá e a Ilha do Marajó, verifica-se a predominância de chuvas máximas noturnas, principalmente no quadrimestre JFMA.

Para áreas entre 100 e $350 \mathrm{~km}$ de distância da costa, o máximo de precipitação ocorre preferencialmente entre $1800 \mathrm{e}$ 2100 UTC. Em algumas regiões entre 350 e 450 km verificou-se que o máximo de precipitação ocorre no início da manhã, como é o caso da parte central do Estado do Pará. Quanto à fração de variância é possível verificar que durante o quadrimestre JFMA, o harmônico diário contribui com valores acima de $90 \%$ para o
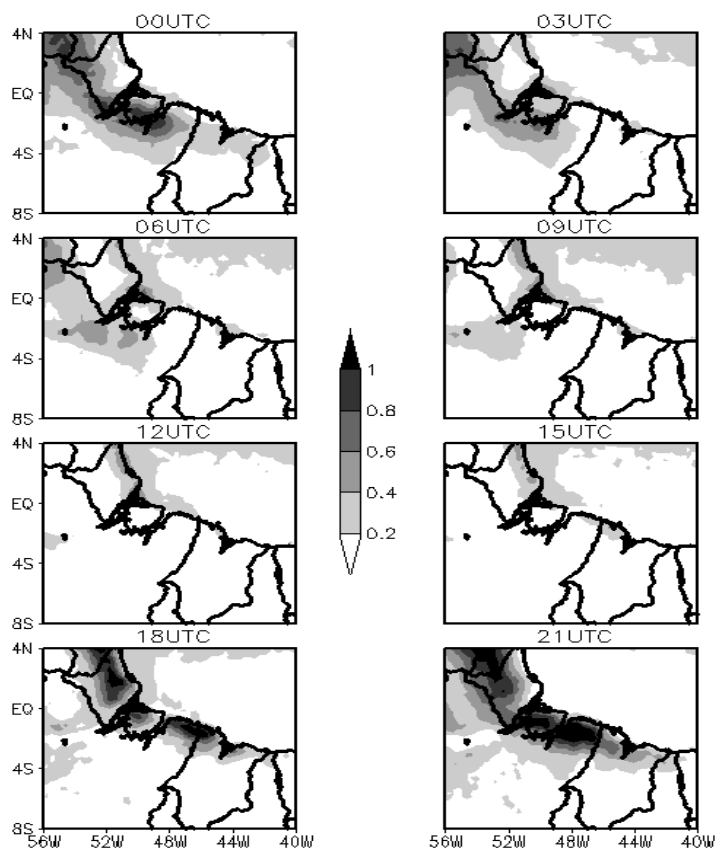

Figura 3 - Semelhante à Figura 2, mas para o quadrimestre MJJA. 
total da precipitação na região entre 100 e $350 \mathrm{~km}$. Ainda durante o quadrimestre JFMA, o harmônico diário explica frações acima de $80 \%$ sobre o oceano, principalmente em regiões mais próxima da costa. No quadrimestre MJJA o ciclo diário explica mais do que $90 \%$ da variância da precipitação em uma linha com orientação NW-SE que se estende desde o Nordeste do Pará até a linha que divide os Estados do Amapá e Pará. Por sua vez, no quadrimestre SOND, o ciclo diário apresenta maior contribuição à variância sobre o Nordeste do Pará e Oeste do Amapá.

Quanto ao ciclo semidiário (Figura 6) verifica-se que a amplitude, a fase e a variância apresentam-se de forma simétrica em relação ao ciclo diário. Regiões onde o ciclo diário é predominante, em termos de alta fração de variância, são coincidentes com baixa fração de variância para o ciclo semidiário e vice-versa. Sobre o oceano, próximo à linha da costa, o ciclo semidiário apresenta fração de variância abaixo de $10 \%$ durante o quadrimestre JFMA. Ao mesmo tempo, essas mesmas regiões, apresentam variância acima de $50 \%$ para os quadrimestres jjas e SOND. As áreas entre 0 e $100 \mathrm{~km}$ e acima de $450 \mathrm{~km}$ da linha da costa apresentam fração de variância acima de $30 \%$ para o ciclo semidiário durante os três quadrimestres.

Por outro lado a região entre 100 e $350 \mathrm{~km}$ é dominada pelo ciclo diário, exceto sobre o Estado do Maranhão. Invariavelmente, a fase do ciclo semidiário ocorre entre 0000 e 0300 UTC na parte mais próxima ( 0 e $100 \mathrm{~km})$ da costa, e preferencialmente às $0000 \mathrm{UTC}$ no restante do continente. A amplitude do ciclo semidiário é maior na parte região continental mais próxima
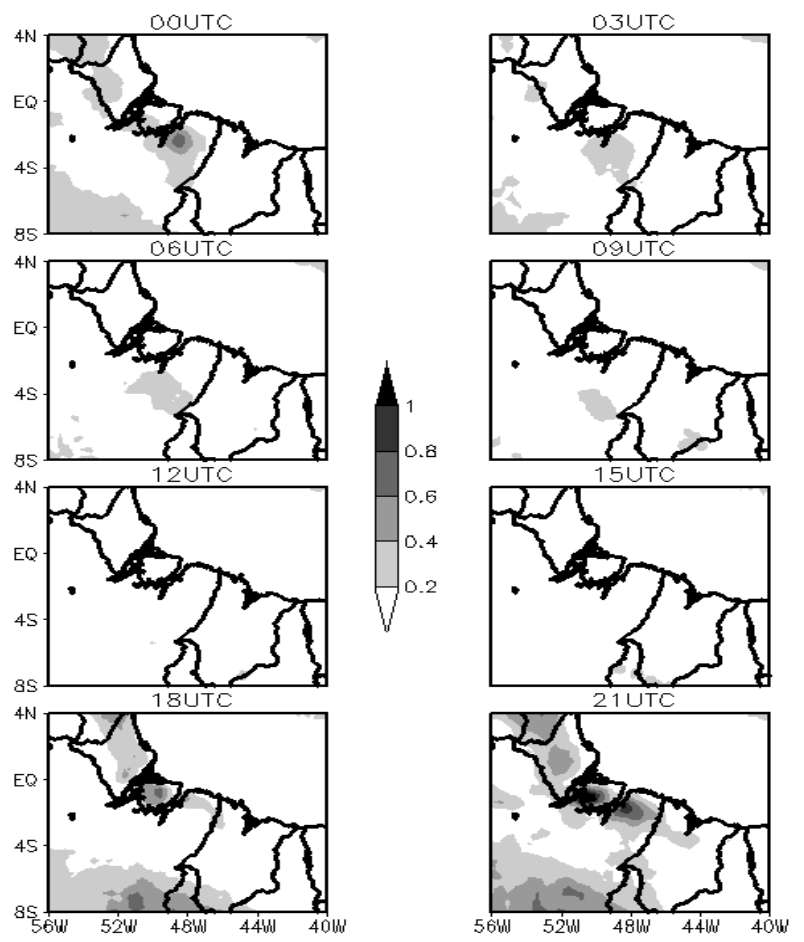

Figura 4 - Semelhante à Figura 2, mas para o quadrimestre SOND. da costa, destacando-se a região Nordeste do Pará durante o quadrimestre JFMA e a porção Norte do Estado do Maranhão.

\section{DISCUSSÃO E CONCLUSÕES}

Dados de precipitação derivados do TRMM têm sido usados em diferentes regiões do globo para identificar as características dos ciclos diário e semidiário de precipitação. Ao mesmo tempo, existe um esforço científico no sentido de verificar as incertezas desses produtos, em relação à precipitação observada através de estações meteorológicas convencionais e automáticas. Quanto à validação especifica do 3B42_V6 para a bacia Amazônica, Santos e Silva et al. (2009a) e Santos e Silva et al. (2011) verificaram que esses dados são bem correlacionado com a precipitação observada através de pluviômetros e por radar; porém, tendem a subestimar em até $50 \%$ os eventos de precipitação mais intensas. Além disso, o 3B42_V6, sistematicamente, aponta o máximo de precipitação às 2100 UTC em áreas continentais no interior da bacia Amazônica, o que também tem sido verificado em outras regiões do globo (Tian et al., 2007).

Apesar dessas incertezas, considera-se que o uso deste produto é muito importante para estudos sobre ciclo diário e semidiário de precipitação, pois cobrem áreas de difícil monitoramento e apresentam amostragem temporal de 3

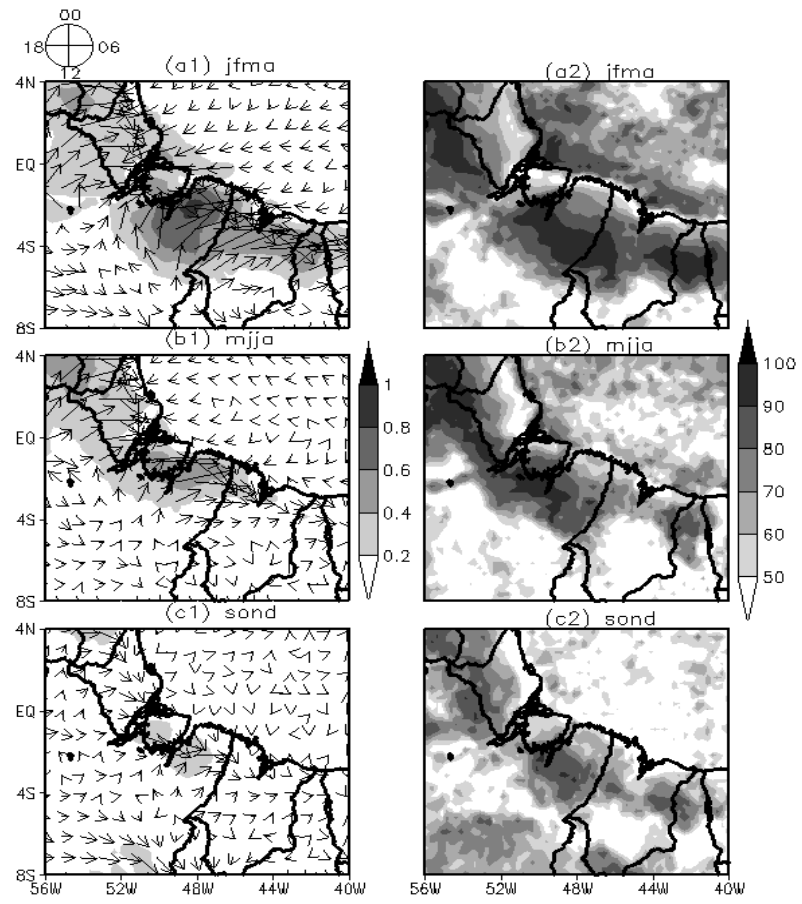

Figura 5 - Amplitude, ângulo de fase e total de variância explicada pelo harmônico diário. A primeira coluna consiste da amplitude em mm h-1 e a fase, representada pelo vetor cuja legenda é mostrada na parte superior da figura. A segunda coluna indica a fração de variância explicada pelo harmônico diário, expressa em porcentagem. 


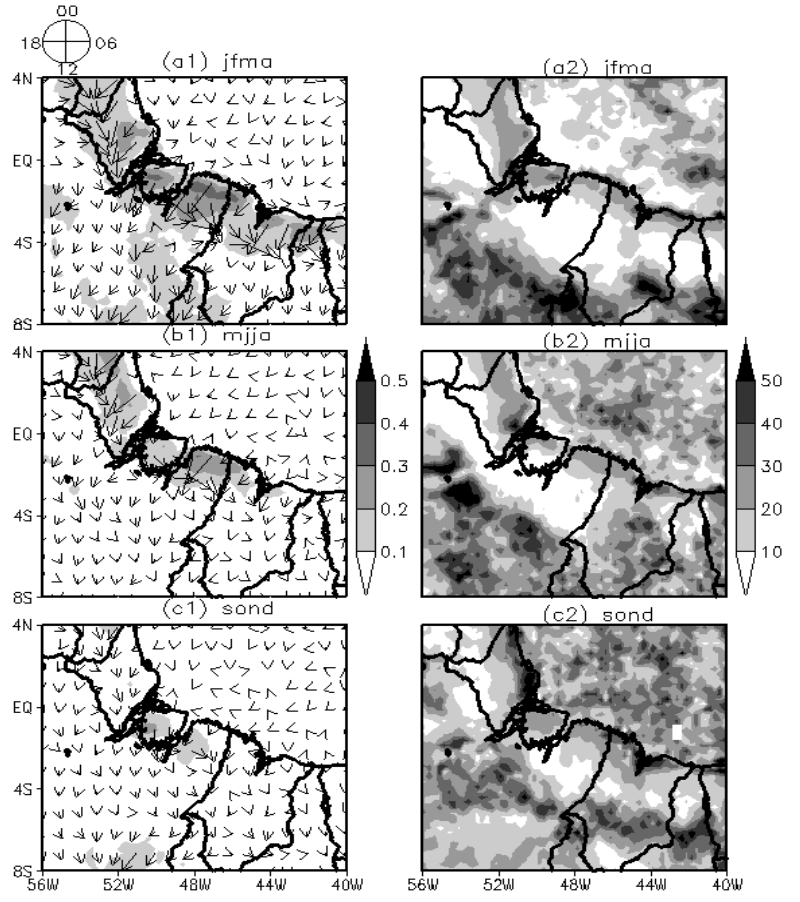

Figura 6 - Semelhante à Figura 5, mas para o ciclo semidiário.

horas para uma grade relativamente fina (em torno de $25 \mathrm{~km}$ ). Portanto, as discussões apresentadas nesta seção devem ser interpretadas levando-se em consideração essas limitações, mas ao mesmo tempo indica-se que este produto tem sido de grande valia para estudos hidrometeorológicos em torno do globo, inclusive havendo propostas de melhorias do 3B42_V6 para uso em diversas aplicações na meteorologia (Rozante et al., 2010).

A partir de dados de 24 estações distribuídas pela bacia Amazônica (não somente no litoral), Angelis et al. (2004) verificaram que na porção Norte da Amazônia a precipitação tende a ocorrer à noite. Em regiões centrais, no Oeste e no Sul, os máximos ocorrem à tarde e o sentido de propagação das chuvas é invariavelmente para Leste. Estes autores notaram uma área relativamente seca em locais situados em torno de $500 \mathrm{~km}$ e verificaram que não há diferenças significativas, em termos sazonais, no horário de máxima precipitação. Esses resultados são consistentes com os apresentados no presente artigo, embora haja uma pequena discrepância na localização da região mais seca (em torno de $450 \mathrm{~km}$ no presente estudo), que pode ser entendida em virtude da distribuição espacial irregular das estações do estudo de Angelis et al. (2004) ou então devido à subjetividade (pois é sabido que este valor depende da latitude) de se fixar a proporção de $1^{\circ}$ para $110 \mathrm{~km}$ na presente pesquisa.

Com dados coletados em diferentes pontos na Colômbia, Poveda et al. (2005) também verificaram a ocorrência de ciclos diário e semidiário bem definidos e com pouca variabilidade sazonal; além disso, mostraram que a amplitude dos ciclos são afetados pela variabilidade interanual, que é função da ocorrência de El Niño e La Niña, sendo o máximo de precipitação mais acentuado em anos de La Niña e também verificaram a modulação da amplitude através de oscilações intrasazonais. Estudar a influência de outras escalas atmosféricas na amplitude, na fase e na variância dos ciclos não é objetivo deste trabalho, mas ressalta-se que é possível a utilização deste mesmo conjunto de dados para esta finalidade. Inclusive sugere-se um estudo sobre o ciclo diário em anos contrastantes como foi 2005 e 2009, anos de seca e cheia, respectivamente.

A partir dos dados analisados nesta pesquisa verificou-se pouca sazonalidade no que concerne à precipitação sobre a costa Norte do Brasil, conforme verificado nas Figuras 5 e 6. Entretanto, em outras regiões, como é o caso da península banhada pelo mar Sul da China observou-se (com os dados do 3B42 V6) mudanças no horário e na localização da máxima precipitação em virtude do regime de monção na região (Li et al. , 2010). Da mesma forma, existe uma marcante sazonalidade na fase do ciclo de precipitação na baia de Bengal, India, conforme apresentado por Roy e Balling Jr (2007), o que também é associado à circulação do tipo monçônica sobre essa região. O Sistema de Monção da América do Sul (SAMS; Jones e Carvalho, 2002) é manifestado na anomalia do campo de vento; portanto, outra possibilidade de estudo é associar possíveis variações sazonais (e intra sazonais) dos ciclos diário e semidiário sobre a América do Sul a partir dos dados do 3B42_V6.

Quanto ao uso da análise harmônica, alguns estudos têm mostrado que o harmônico diário e o harmônico semidiário contribuem conjuntamente para algo em torno de 60 a $90 \%$ do total da variância da precipitação no período de 24 horas, sendo o ciclo diário o principal modo de variabilidade (Dai, 2001), apesar de o ciclo semidiário estar presente sistematicamente em diversas regiões do globo, principalmente regiões costeiras (Yang et al., 2008). Os resultados apresentados na seção 3.2 ratificam esses estudos prévios, embora em algumas regiões, especialmente o Nordeste do Pará, o harmônico diário e o semidiário contribuam para quase $100 \%$ da variância da precipitação em 24 horas, ao mesmo tempo em que apresentam amplitudes acima de $1 \mathrm{~mm} \mathrm{~h}^{-1}$, trata-se, portanto, de uma região em que os mecanismos de brisa e convecção local (mecanismos Continental 1 e Continental 3, vide seção 1.1) devam atuar sinergeticamente conforme proposto por Yang e Smith (2006).

Por fim, alguns estudos revelam que pequenas variações no horário de máxima precipitação podem acarretar mudanças significativas no total de precipitação sobre uma região, como é o caso do Japão (Fugibe et al., 2006). Portanto, a análise de tendência das características do ciclo diário e do ciclo semidiário são temas importantes para o entendimento de mudanças e variabilidade do clima. Entretanto, a série histórica dos dados 
provenientes do projeto TRMM, e de outros produtos de chuva estimada por técnicas de satélites, ainda não são suficientes para analisar a tendência de precipitação. Mas servem como fonte para avaliação e calibração de modelos de circulação geral da atmosfera ou modelos estocásticos, que em última análise podem provir simulações de diferentes cenários climáticos futuros.

\section{REFERÊNCIAS BIBLIOGRÁFICAS}

ANGELIS, C.F.; McGREGOR, G.R.; KIDD, C. Diurnal cycle of rainfall over the Brazilian Amazon. Climate Research, v. 26, p. 139-149, 2004.

BARBOSA, R.L.; OYAMA, M.D.; MACHADO, L.A.T. Climatologia das perturbações convectivas iniciadas na costa Norte do Brasil. Revista Brasileira de Meteorologia, v. 21, p. 107-117, 2006.

BOCCIPPIO, D.J.; KOSHAK, W.; BLAKESLEE, R.J. Performance assessment of the optical transient detector and lightning imaging sensor. Part 1: Predicted diurnal variability. Journal of Atmospheric and Oceanic Technology, v. 19, p. 1318-1332, 2002.

CHEN, J. E WANG, J. Diurnal variation of surface thermodynamic fields on the island of Hawaii. Monthly Weather Review, v. 122, p. 2125-2138, 1994.

COHEN, J.C.P.; SILVA DIAS, M. A. F.; NOBRE, C. A. Environmental conditions associated with Amazonian squall lines: a case study. Monthly Weather Review, v.123, p. 3163-3174, 1995.

DAI, A. E WANG, J. Diurnal and semidiurnal tides in global surface pressure fields. Journal of the Atmospheric Science, v.56, p. 3874-3891, 1999.

DAI, A. Global Precipitation and Thunderstorm Frequencies. Part II: Diurnal Variations. Journal of Climate, v. 14, p. 1112-1128, 2001.

DOUGLAS, M.A.; VALDEZ-MANZANILLA, A.; CUETO, R.G. Diurnal variation and horizontal extent of the low-level jet over the northern Gulf of California. Monthly Weather Review, v. 126, p. 2017-2025, 1998.

FUJIBE, F.; YAMAZAKI, N.; KOBAYASHI, K. Long-term changes in the diurnal precipitation cycles in Japan for 106 years (1898-2003). Journal of the Meteorological Society of Japan, v. 84, p. 311-317, 2006.

GRAY, W.M. AND JACOBSON, R.W. Diurnal variation of deep cumulus convection. Monthly Weather Review, v. 105, p. 1171-1188, 1977.

HARTMANN, D.L.; KOWALEWSKY, K.J.; MICHELSEN, M.L. Diurnal variations of outgoing longwave radiation and albedo from ERBE Scanner data. Journal of Climate, v. 4, p. 598-617, 1991.
HUFFMAN, G.J. et al. The TRMM Multisatellite Precipitation Analysis (TMPA): quasi-global, multiyear, combinedsensor precipitation estimative at fine scales. Journal of Hydrometeorology, v. 8, p. 38-55, 2007.

JONES, C.; CARVALHO, L.M.V. Active and break phases in the South American monsoon system. Journal of Climate, v. 15, p. 905-915, 2002.

LI, W.; LUO, C.; WANG, D.; LEI, T. Diurnal variation of precipitation over the South China Sea. Meteorology and Atmospheric Physics, v. 109, p. 33-46, 2010.

KOUSKY, V.E. Diurnal rainfall variation in northeast Brazil. Monthly Weather Review, v. 108, p. 488-498, 1980.

LINDZEN, R.S. Effect of daily variations of cumulonimbus activity on the atmospheric semidiurnal tide. Monthly Weather Review, v. 106, p. 526-533, 1978.

MACHADO, L.A.T.; DUVEL, J.P.; DESBOIS, M. Diurnal variations and modulation by easterly waves of the size distribution of convective cloud clusters over West Africa and the Atlantic Ocean. Monthly Weather Review, v. 121, p.37-49, 1993.

MAPES, B.E.; WARNER, T.; XU, M. Diurnal patterns of rainfall in Northwestern South America. Part III: diurnal gravity waves and nocturnal convection offshore. Monthly Weather Review, v. 131,p. 830-844, 2003 a.

MAPES, B.E.; WARNER, T.; XU, M.; NEGRI, A.J. Diurnal patterns of rainfall in Northwestern South America. Part I: observations and context. Monthly Weather Review, v. 131, p. 799-812, 2003 b.

MARENGO, J.; NOBRE, C. Clima da região amazônica. In: CAVALCANTI, I.F.A. (Org.). Tempo e Clima do Brasil São Paulo: Oficina de Textos, 2009, p.198-212.

MARENGO, J.A.; LIEBMANN, B.; KOUSKY, V.E.; FILIZOLA, N.P.; WAINER, I.C. Onset and end of the rainy season in brazilian Amazon Basin. Journal of Climate, v. 14, p. 833-852, 2001.

OGURA, Y. E.; TAKAHASHI T. Numerical simulation of the cycle of a thunderstorm cell. Monthly Weather Review, v. 99, p. 895-911, 1971.

PIELKE, R. A. A three-dimensional numerical model of Sea breezes over South Florida. Monthly Weather Review. v. 102, p. 115-139, 1974.

POVEDA, G.; MESA, O.; SALAZAR, L.; ARIAS, P.; MORENO, H.; VIEIRA, S.; AGUDELO, P.; TORO, V.; ALVAREZ, J. The diurnal cycle of precipitation in the tropical Andes of Colombia. Monthly Weather Review, v. 133, p. 228-240, 2005.

RANDALL, D.A.; HARSHVARDHAN; DAZLICH, D.A. Diurnal variability of the hydrologic cycle in a General Circulation Model. Journal of Atmospheric Science, v. 48, p. 40-62, 1991. 
ROY, S.S.; BALLING JR, R.C. Diurnal variations in summer season precipitation in India. International Journal of Climatology, v. 27, p. 969-976, 2007.

ROZANTE, J.R.; MOREIRA, D.S.; GONÇALVES, L.G.G.; VILA, D.A. Combining TRMM and surface observations of precipitation: technique and validation over South America. Weather and Forecasting, v. 25, p. 885-894, 2010.

SANTOS E SILVA, C.M.; FREITAS S.R.; GIELOW R.; BARROS, S. Evaluation of high-resolution precipitation estimates over the Amazon Basin. Atmospheric Science Letters, v.10, p. 273-378, 2009a.

SANTOS E SILVA, C.M.; FREITAS, S.R.; GIELOW, R. Ciclo diário de precipitação estimada através de um radar banda S e pelo algoritmo 3B42_V6 do projeto TRMM durante a estação chuvosa de 1999 no Sudeste da Amazônia. Revista Brasileira de Meteorologia, v.26, p. 95 - 108, 2011

SILVA DIAS, P.L.; BONATTI, J.P.; KOUSKY, V.E. Diurnally forced tropical tropospheric circulation over South America. Monthly Weather Review, v. 115, p. 1465-1478, 1987.

SIMPSON, J.; ADLER, R.F.; NORTH, G.R. A proposed Tropical Rainfall Measuring Mission Satellite. Bulletin of the American Meteorological Society, v. 69, p. 278-295, 1988.

SOUZA, E.B.; ROCHA, E.P. Diurnal variation of rainfall in Bragança-PA (Eastern Amazon) during rainy season: mean characteristics and extreme events. Revista Brasileira de Meteorologia, v. 21, p-142-152, 2006.

SUI, C.H.; LAU, K.M.; TAKAYABU, Y.N.; SHORT, D.A. Diurnal variation in tropical oceanic cumulus convection during TOGA COARE. Journal of Atmospheric Science, v. 54, p. 639-655, 1997.

TIAN, Y.; PETERS-LIDARD, C.D.; CHOUDHURY, B.J.; GARCIA, M. Multitemporal analyses of TRMM-based satellite precipitation products for land data assimilations applications. Journal of Hydrometeorology, v. 8, p. 1165-1183, 2007.
TRIPOLI, G.J. An explicit three-dimensional nonhydrostatic numerical simulation of a tropical cyclone. Meteorology and Atmospheric Physics, v. 49, p. 229-254, 1992.

TRIPOLI, G.J. E COTTON, W.R. Numerical study of an observed orogenic Mesoscale Convective System. Part 2: analysis of governing dynamics. Monthly Weather Review, v. 117, p. 305-328, 1989.

WALLACE, J.M. Diurnal variations in precipitation and thunderstorm frequency over the conterminous United States. Monthly Weather Review, v. 103, p. 406-419, 1975.

WALLACE,J.M. E PATTON, D.B. Diurnal temperature variations: surface to 25 kilometers. Monthly Weather Review, v. 98, p. 548-552, 1970.

WARNER, T.T.; MAPES, B.E.; XU, M. Diurnal patterns of rainfall in Northwestern South America.Part II: model simulations. Monthly Weather Review, v. 131, p. 813829, 2003.

WEBSTER, P.J.; CLAYSON, C.A.; CURRY, J.A. Clouds, radiation, and the diurnal cycle of sea surface temperature in the tropical Western Pacific. Journal of Climate, v. 9, p. 1712-1730, 1996.

WILKS, D.S. Statistical Methods in the Atmospheric Sciences: An Introduction. Academic Press: San Diego, CA, 2006.

XU, K.M.; EMANUEL, K.A. Is a Tropical atmosphere conditionally unstable? Monthly Weather Review, v.117, p. 1471-1479, 1989.

YANG, S. E SMITH, E.A. Mechanism for diurnal variability of global tropical rainfall observed for TRMM. Journal of Climate, v. 19, p. 5190-5226, 2006.

YANG, S.; KUO, K.; SMITH, E. Persistent nature of secondary diurnal modes of precipitation over oceanic and continental regimes. Journal of Climate, v. 21, p. 4115-4131, 2008. 